\title{
Improved plant transformation vectors for fluorescent protein tagging
}

\author{
Silin Zhong • Zhefeng Lin • Rupert G. Fray • \\ Don Grierson
}

Received: 30 January 2008/Accepted: 10 June 2008/Published online: 2 July 2008

(C) The Author(s) 2008

\begin{abstract}
Fluorescent protein labelling technologies enable dynamic protein actions to be imaged in living cells and can also be used in conjunction with other methods such as Forster resonance energy transfer and biomolecular fluorescence complementation. In this report, we describe the generation of a series of 23 novel GATEWAY-compatible vectors based on pGreenII and pDH51 backbones with the latest fluorescent protein tags (Cerulean, EGFP and Venus) and the choice of three in planta selection markers. These vectors can be obtained from the Nottingham Arabidopsis Stock Centre (N9819-N9846) and should be a powerful tool box for transgenic research in plants.
\end{abstract}

Keywords BiFC . Binary vector . Fluorescent protein $\cdot$ FRET $\cdot$ pDH51 $\cdot$ pGreen $\cdot$ GATEWAY

$\begin{array}{ll}\begin{array}{l}\text { Abbreviations } \\ \text { BiFC }\end{array} & \begin{array}{l}\text { Biomolecular fluorescence } \\ \text { complementation } \\ \text { Cauliflower mosaic virus 35S } \\ \text { promoter/terminator }\end{array} \\ \text { CaMV 35S pro/ter }\end{array}$

Accession numbers: AM773751-AM773753; AM779183AM779184; AM773753-AM884388.

S. Zhong · Z. Lin · R. G. Fray · D. Grierson $(\bowtie)$

Plant Sciences Division, School of Biosciences, University of Nottingham, Sutton Bonington Campus, Loughborough LE12 5RD, UK

e-mail: Donald.Grierson@Nottingham.ac.uk

$\begin{array}{ll}\text { CFP } & \text { Cyan fluorescent protein } \\ \text { GFP } & \text { Green fluorescent protein } \\ \text { YFP } & \text { Yellow fluorescent protein } \\ \text { FRET } & \text { Forster resonance energy } \\ & \text { transfer } \\ \text { MCS } & \text { Multi-cloning site } \\ \text { mRFP1 } & \begin{array}{l}\text { Monomeric red fluorescent } \\ \text { protein1 }\end{array}\end{array}$

\section{Introduction}

The discovery and cloning of the fluorescent protein from the bioluminescent jellyfish Aequorea victoria have revolutionized studies in cell biology by enabling the dynamic monitoring of protein localization in the living cell using fluorescent microscopy (Prasher et al. 1992). The application of fluorescent protein in higher plants was greatly improved when a cryptic intron within the GFP coding sequence was removed (Haseloff et al. 1997). Because of their intrinsic fluorescence ability and minimal toxicity, fluorescent proteins have been widely used as noninvasive markers in many living organisms. Extensive mutagenesis screens had been carried out and numerous GFP variants with distinct fluorescence characteristic had been generated (Shaner et al. 2005). A further development was the demonstration that protein-protein interaction in the living cell could be detected by Forster resonance energy transfer (FRET) using fluorescent proteins with 
different fluorescence profiles (Gordon et al. 1998; Periasamy and Day 1999). An alternative technique known as biomolecular fluorescence complementation (BiFC) has also been developed (Hu et al. 2002). $\mathrm{BiFC}$ is based on the ability of two non-fluorescent fragments of a fluorescent protein to re-constitute a functional fluorescent complex. Thus, when the two fragments are brought together by the interaction of two target proteins fused to the fragments, fluorescent signal can be observed, which serves as an indicator of the target protein interaction.

Many GATEWAY binary vector systems have been generated to enable in planta expression of recombinant proteins fused to fluorescent tags (Karimi et al. 2002; Bracha-Drori et al. 2004; Earley et al. 2006). However, the large binary plasmids are difficult to modify when a new variety of the fluorescent protein becomes available. For instance, it has been shown that the YFP variant Venus (F46L/F64L/M153T/V163A/S175G mutated EYFP) outperformed the obsolete enhanced YFP (EYFP) in both FRET and BiFC experiments (Nagai et al. 2002; Shyu et al. 2006), whilst a brighter enhanced CFP (ECFP) variant Cerulean (S72A/Y145A/H148D mutated ECFP) has a single exponential lifetime that is ideal for FLIM-FRET measurement (Rizzo et al. 2004). However, no plant transformation vector has yet been reported to utilize these new FPs, possibly due to the difficulties in manipulating the large binary plasmids. Hellens and his co-workers addressed this issue by generating a small and easy-to-modify binary vector known as pGreen, which is only $3 \mathrm{~kb}$ in size and requires a separate helper plasmid (pSOUP) to support its replication in Agrobacterium (Hellens et al. 2000).

In this report we describe the generation of a series of GATEWAY-compatible plant transformation vectors based on the pDH51 (Pietrzak et al. 1986) and pGreenII backbones with three new fluorescent protein tags (Cerulean, EGFP and Venus) suitable for protein localization, FRET and BiFC experiments.

\section{Methods, results and discussion}

We first PCR amplified the cDNAs of three GFP variants (Cerulean, EGFP, and Venus) and cloned them into the BamHI and SalI linearized pDH51 plasmid, which contains a cauliflower mosaic virus
$35 \mathrm{~S}$ promoter and terminator but without T-DNA borders. The attR1-Cm ${ }^{\mathrm{R}}$-ccdB-attR2 GATEWAY cassette (Invitrogen) was then inserted into the aforementioned pDH51 plasmids linearized by SmalI and XhoI. For the BiFC vectors, the $\mathrm{N}$ - and $\mathrm{C}$-termini of the YFP variant Venus (referred to as YFPn and YFPc) were cloned into pDH51 and the resulting plasmids were converted to GATEWAY destination vectors. For plant transformation, two sets of GATEWAY vectors were constructed using pGreenII plasmids with three types of in planta selection markers. The first set contains a cauliflower mosaic virus $35 \mathrm{~S}$ promoter to express the transgene, whilst the second series has a multiple cloning site (MCS) instead of the promoter. To generate the first set of vectors, the expression cassettes from the aforementioned pDH51 vectors were released by either EcoRI/ HindIII or KpnI digestion and cloned to three pGreenII plasmids pGreenII0029, pGreenII0229 and pGreenII0179 (BASTA, kanamycin, hygromycin resistance, respectively).

To demonstrate the utility of these new fluorescent proteins, sub-cellular localization studies were carried out to investigate several components in the ethylene signalling pathway using biolistic-mediated transformation of onion cells as described in Scott et al. (1999). Due to the small size of our pDH51based vectors, $1 \mu \mathrm{g}$ of plasmid DNA, instead of $10 \mu \mathrm{g}$, was coated onto gold particles (sphere 0.8-1.5 $\mu \mathrm{m}$, AlfaAesar) and bombarded into onion epidermal peels. We show here that both the tomato ethylene receptor LeETR4 and the downstream positive regulator LeEIN2 (Tieman and Klee 1999; Zhu et al. 2006) were targeted to the endoplasmic reticulum, as was the ER-YFP control (Fig. 1a-c). In a co-localization experiment, GREEN-RIPE (GR-YFP), whose ectopic expression caused reduced ethylene responsiveness (Barry and Giovannoni 2006), was found in the Golgi with the marker GONST1-CFP and did not co-localized with the ER targeted AtRAN1-CFP (Fig. 1d-f). The pGreenbased binary vector pGBPGWG has also been used to express a methyltransferase (At4G10760) fused to GFP in the stably transformed Arabdiopsis plants (Fig. 1g). In addition, these vectors have been used in a separated study to demonstrate the localization and protein-protein interaction of tomato ethylene receptor NEVER-RIPE and the LeCTR proteins (Zhong et al. 2008). 

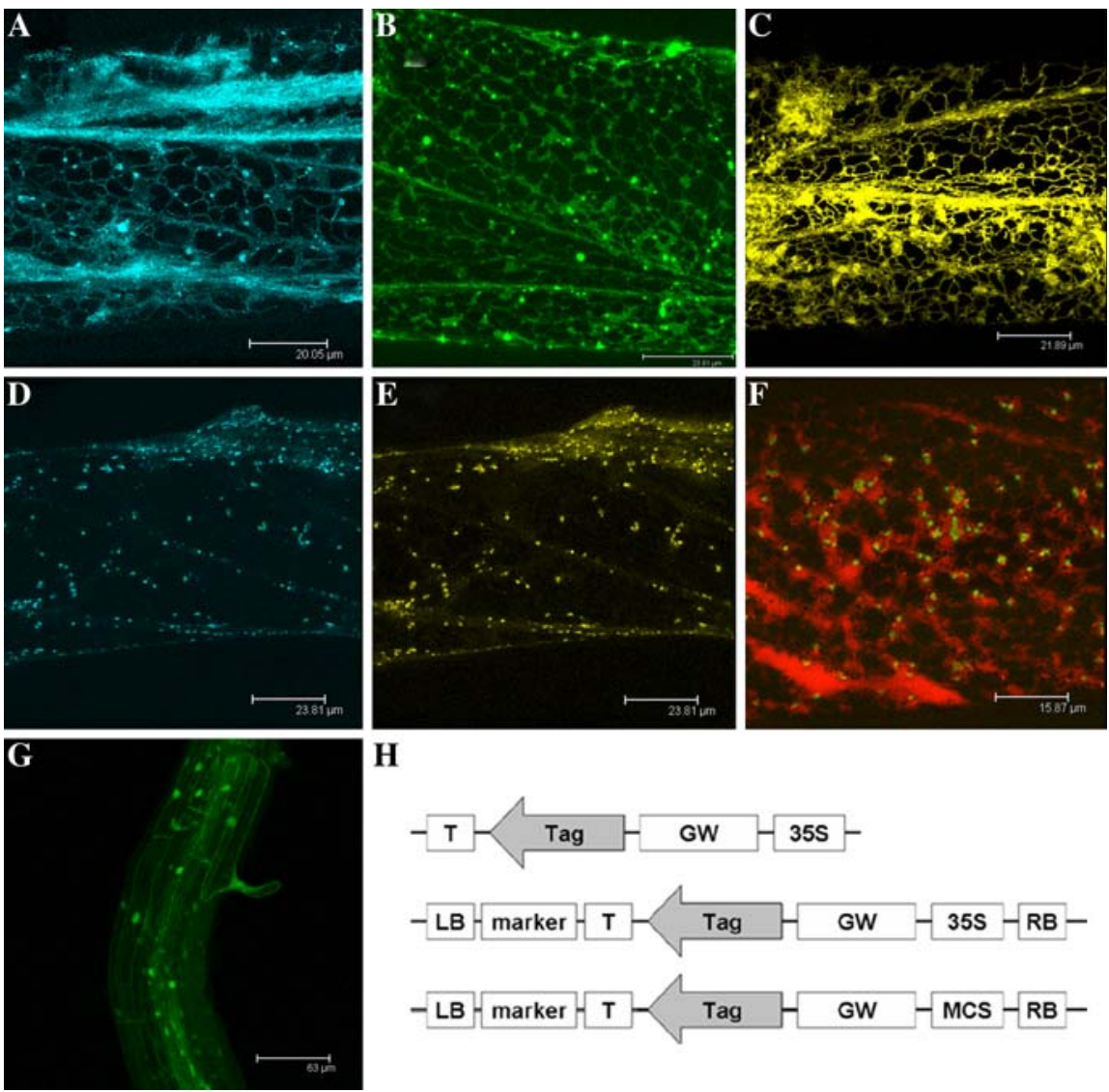

H

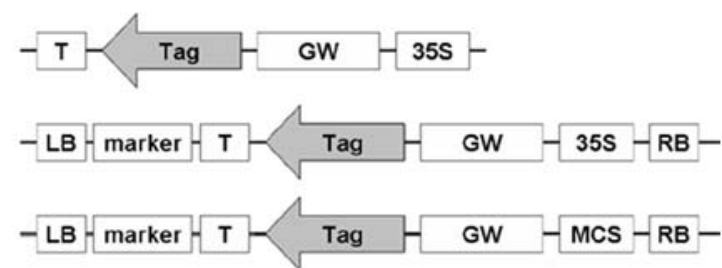

Fig. 1 Transient expression of fluorescent proteins in onion epidermal cells. (a) Confocal images of an onion epidermal cell expressing the tomato sub-family II ethylene receptor LeETR4 fused to Cerulean (CFP). (b) Tomato ethylene signalling component LeEIN2-GFP. (c) Venus (YFP) fused to N-terminal ER targeting sequence and C-terminal ER retention signal (HDEL) expressed in an onion cell as the ER control. (d and e) A single onion cell expressing Arabidopsis protein GONST1 fused to Cerulean as a Golgi marker (blue image, d) and GREEN-RIPE fused to Venus (yellow image, e).

In this work, we describe a series of plant transformation vectors that are compatible with the GATEWAY cloning system to enable expression of proteins fused with the latest variants of fluorescent protein tags (Fig. 1h). The CFP variant Cerulean used in these vectors has been estimated to be 1.5 times brighter than the original ECFP and has the highest quantum yield among all CFP variants (Rizzo et al. 2004). Additionally, it is the only CFP with a single exponential fluorescence lifetime, which is crucial for FLIM-FRET experiments. The YFP variant Venus was chosen to replace the earlier EYFP, which is sensitive to $\mathrm{pH}$ and matures slowly (Nagai et al. (f) The Arabidopsis ethylene signalling component RAN1 fused to Cerulean (false colour red) co-expressed with the GREEN-RIPE tagged with Venus (false colour green). (g) Stably transformed Arabidopsis expressing a methyltransferase fused to GFP. (h) Schematic diagrams of the vectors: pDH51GW-FP (top), pGreenII-35S-GW-FP (middle), pGreenII-MCSGW-FP (bottom). T, terminator; GW, attR cassette; MCS, multi-cloning site; LB/RB, T-DNA border; marker, in planta selection genes. Scale bars: a $20 \mu \mathrm{m}$, b $24 \mu \mathrm{m}$, c $22 \mu \mathrm{m}$, d $24 \mu \mathrm{m}$, e $24 \mu \mathrm{m}, \mathbf{f} 16 \mu \mathrm{m}, \mathbf{g} 61 \mu \mathrm{m}$

2002). We generated the BiFC vectors based on the Venus $\mathrm{N}$ - and $\mathrm{C}$-terminal fragments as it has been shown that they could increase the BiFC efficiency up to 13-fold compared to the original fragments derived from EYFP (Shyu et al. 2006).

Biolistic-mediated transient expression is a simple and efficient approach to study in vivo protein localization and interaction. However, it requires microgram quantities of plasmid DNA and large scale preparation of traditional binary plasmids is costly and time consuming. The first set of vectors is based on the minimal pDH51 plasmid and is less than $6 \mathrm{~kb}$ in size. Therefore, these plasmids are ideal for particle 
Table 1 List of vectors for fluorescent protein tagging

\begin{tabular}{|c|c|c|c|c|}
\hline NASC code & Accession number & Name & Features & Bacterial selection \\
\hline N9821 & AM884371 & pGBPGWC & $35 \mathrm{~S}$ promoter, CFP tag, BASTA & Kan, Cm \\
\hline N9822 & AM884372 & pGBPGWG & $35 \mathrm{~S}$ promoter, GFP tag, BASTA & $\mathrm{Kan}, \mathrm{Cm}$ \\
\hline N9823 & AM884373 & pGBPGWY & $35 \mathrm{~S}$ promoter, YFP tag, BASTA & $\mathrm{Kan}, \mathrm{Cm}$ \\
\hline N9824 & AM884374 & pGHPGWC & $35 \mathrm{~S}$ promoter, CFP tag, Hygromycin & Kan, Cm \\
\hline N9825 & AM884375 & pGHPGWG & $35 \mathrm{~S}$ promoter, GFP tag, Hygromycin & Kan, Cm \\
\hline N9826 & AM884376 & pGHPGWY & 35S promoter, YFP tag, Hygromycin & Kan, Cm \\
\hline N9827 & AM884377 & pGKPGWC & 35S promoter, CFP tag, Kanamycin & Kan, Cm \\
\hline N9828 & AM884378 & pGKPGWG & 35S promoter, GFP tag, Kanamycin & $\mathrm{Kan}, \mathrm{Cm}$ \\
\hline N9829 & AM884379 & pGKPGWY & 35S promoter, YFP tag, Kanamycin & $\mathrm{Kan}, \mathrm{Cm}$ \\
\hline N9830 & AM884380 & pGKGWY & No promoter, YFP tag, Kanamycin & Kan, Cm \\
\hline N9831 & AM884381 & pGKGWG & No promoter, GFP tag, Kanamycin & Kan, Cm \\
\hline N9832 & AM884382 & pGKGWC & No promoter, CFP tag, Kanamycin & Kan, Cm \\
\hline N9833 & AM884383 & pGHGWC & No promoter, CFP tag, Hygromycin & Kan, Cm \\
\hline N9834 & AM884384 & pGHGWG & No promoter, GFP tag, Hygromycin & Kan, Cm \\
\hline N9835 & AM884385 & pGHGWY & No promoter, YFP tag, Hygromycin & Kan, Cm \\
\hline N9836 & AM884386 & pGBGWC & No promoter, CFP tag, BASTA & Kan, Cm \\
\hline N9837 & AM884387 & pGBGWG & No promoter, GFP tag, BASTA & $\mathrm{Kan}, \mathrm{Cm}$ \\
\hline N9838 & AM884388 & pGBGWY & No promoter, YFP tag, BASTA & Kan, Cm \\
\hline N9839 & AM773751 & pDH51-GW-CFP & 35Spro-GW-Cerulean-Ter & $\mathrm{Amp}, \mathrm{Cm}$ \\
\hline N9840 & AM773753 & pDH51-GW-GFP & 35Spro-GW-EGFP-Ter & $\mathrm{Amp}, \mathrm{Cm}$ \\
\hline N9841 & AM773752 & pDH51-GW-YFP & 35Spro-GW-Venus-Ter & Amp, $\mathrm{Cm}$ \\
\hline N9842 & AM779183 & pDH51-GW-YFPn & 35Spro-GW-YFPn-Ter & $\mathrm{Amp}, \mathrm{Cm}$ \\
\hline N9843 & AM779184 & pDH51-GW-YFPc & 35Spro-GW-YFPc-Ter & $\mathrm{Amp}, \mathrm{Cm}$ \\
\hline N9844 & N/A & pDH51-mRFP1 & 35Spro-mRFP1-Ter (BiFC control) & Amp \\
\hline N9845 & N/A & pDH51-YFPn & 35Spro-YFPn-Ter (BiFC control) & Amp \\
\hline N9846 & N/A & pDH51-YFPc & 35Spro-YFPc-Ter (BiFC control) & Amp \\
\hline
\end{tabular}

The GATEWAY destination vector plasmids are propagated in DB3.1 cells with the antibiotic chloramphenicol to select the Cm ${ }^{\mathrm{R}}$ gene in the GATEWAY cassette. The three selectable marker genes shown in the Features section are based on the pGreenII backbone. Kan, kanamycin; Amp, ampicillin; Cm, chloramphenicol. GW, GATEWAY cassette with attR1 and attR2 recombination sites; 35Spro and Ter, cauliflower mosaic virus 35S promoter and terminator; YFPn, N-terminus of Venus (aa 1-154); YFPc, Cterminus of Venus (aa 155-238)

bombardment experiments and $1 \mu \mathrm{g}$ spin column miniprep plasmid DNA was routinely used with success in our experiments. Once the protein localization and interaction has been tested in a pilot experiment using transient expression, stable plant transformation could be carried out using the second set of vectors based on the pGreenII plasmids. For in vivo protein interaction studies, plants expressing recombinant GFP proteins can be transformed with the pEarleyGate or pGWB vectors with various epitope tags (Earley et al. 2006; Nakagawa et al. 2007), which enables immuno-precipitation experiment to be carried out without raising customer antibodies.
Detailed information about the vectors can be found at the web site of the Nottingham Arabidopsis Stock Centre (NASC) under the "Nottingham pGreen plasmid vectors" collection, and the full sequence with annotation can also be downloaded from the EMBL nucleotide sequence database using their accession numbers (Table 1). All vectors are available from NASC and they should be a valuable tool for transgenic plant research.

Acknowledgements We thank A. Miyawaki for the Venus clone, R. Tsien for the mRFP1, P. Mullineaux for the pGreen plasmids. Part of the work was funded by the Biotechnology and Biological Sciences Research Council. 
Open Access This article is distributed under the terms of the Creative Commons Attribution Noncommercial License which permits any noncommercial use, distribution, and reproduction in any medium, provided the original author(s) and source are credited.

\section{References}

Barry CS, Giovannoni JJ (2006) Ripening in the tomato Greenripe mutant is inhibited by ectopic expression of a protein that disrupts ethylene signaling. Proc Natl Acad Sci USA 103:7923-7928. doi:10.1073/pnas.0602319103

Bracha-Drori K, Shichrur K, Katz A, Oliva M, Angelovici R, Yalovsky S et al (2004) Detection of protein-protein interactions in plants using bimolecular fluorescence complementation. Plant J 40:419-427. doi:10.1111/j. 1365-313X.2004.02206.x

Earley KW, Haag JR, Pontes O, Opper K, Juehne T, Song K et al (2006) Gateway-compatible vectors for plant functional genomics and proteomics. Plant J 45:616-629. doi: 10.1111/j.1365-313X.2005.02617.x

Gordon GW, Berry G, Liang XH, Levine B, Herman B (1998) Quantitative fluorescence resonance energy transfer measurements using fluorescence microscopy. Biophys J 74:2702-2713

Haseloff J, Siemering KR, Prasher DC, Hodge S (1997) Removal of a cryptic intron and subcellular localization of green fluorescent protein are required to mark transgenic Arabidopsis plants brightly. Proc Natl Acad Sci USA 94:2122-2127. doi:10.1073/pnas.94.6.2122

Hellens RP, Edwards EA, Leyland NR, Bean S, Mullineaux PM (2000) pGreen: a versatile and flexible binary Ti vector for Agrobacterium-mediated plant transformation. Plant Mol Biol 42:819-832. doi:10.1023/A:1006496308160

Hu C-D, Chinenov Y, Kerppola TK (2002) Visualization of interactions among bZIP and Rel family proteins in living cells using bimolecular fluorescence complementation. Mol Cell 9:789-798. doi:10.1016/S1097-2765(02)00496-3

Karimi M, Inze D, Depicker A (2002) GATEWAY vectors for Agrobacterium-mediated plant transformation. Trends Plant Sci 7:193-195. doi:10.1016/S1360-1385(02)02251-3

Nagai T, Ibata K, Park ES, Kubota M, Mikoshiba K, Miyawaki A (2002) A variant of yellow fluorescent protein with fast and efficient maturation for cell-biological applications. Nat Biotechnol 20:87-90. doi:10.1038/nbt0102-87

Nakagawa T, Kurose T, Hino T, Tanaka K, Kawamukai M, Niwa $Y$ et al (2007) Development of series of gateway binary vectors, pGWBs, for realizing efficient construction of fusion genes for plant transformation. J Biosci Bioeng 104:34-41. doi:10.1263/jbb.104.34

Periasamy A, Day RN (1999) Visualizing protein interactions in living cells using digitized GFP imaging and FRET microscopy. Methods Cell Biol 58:293-314. doi:10.1016/ S0091-679X(08)61962-7

Pietrzak M, Shillito RD, Hohn T, Potrykus I (1986) Expression in plants of two bacterial antibiotic resistance genes after protoplast transformation with a new plant expression vector. Nucleic Acids Res 14:5857-5868. doi:10.1093/nar/ 14.14.5857

Prasher DC, Eckenrode VK, Ward WW, Prendergast FG, Cormier MJ (1992) Primary structure of the Aequorea victoria green-fluorescent protein. Gene 111:229-233. doi:10.1016/0378-1119(92)90691-H

Rizzo MA, Springer GH, Granada B, Piston DW (2004) An improved cyan fluorescent protein variant useful for FRET. Nat Biotechnol 22:445-449. doi:10.1038/nbt945

Scott A, Wyatt S, Tsou PL, Robertson D, Stromgren Allen N (1999) Model system for plant cell biology: GFP imaging in living onion epidermal cells. Biotechniques 26:1125-1132

Shaner NC, Steinbach PA, Tsien RY (2005) A guide to choosing fluorescent proteins. Nat Methods 2:905-909. doi: $10.1038 /$ nmeth819

Shyu YJ, Liu H, Deng X, Hu CD (2006) Identification of new fluorescent protein fragments for bimolecular fluorescence complementation analysis under physiological conditions. Biotechniques 40:61-66

Tieman DM, Klee HJ (1999) Differential expression of two novel members of the tomato ethylene-receptor family. Plant Physiol 120:165-172. doi:10.1104/pp. 120.1.165

Zhu H, Zhu B, Shao Y, Wang X, Lin X, Xie Y et al (2006) Tomato fruit development and ripening are altered by the silencing of LeEIN2 gene. J Integr Plant Biol 48:14781485. doi:10.1111/j.1744-7909.2006.00366.x

Zhong S, Lin Z, Grierson D (2008) Tomato ethylene receptorCTR interactions: visualization of NEVER-RIPE interactions with multiple CTRs at the endoplasmic reticulum. J Exp Bot 59:965-972. doi:10.1093/jxb/ern021 